\title{
Ist Leiden nicht Sache der Medizin?
}

\section{Hans Stalder}

Prof. Dr., Mitglied der Redaktion

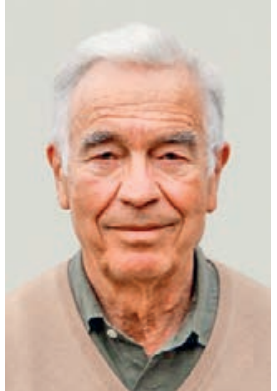

The relief of suffering, it would appear, is considered one of the primary ends of medicine by patients and lay persons, but not by the medical profession [1].

Die Ärztekammer der FMH hat es abgelehnt, die Richtlinien «Umgang mit Sterben und Tod» der Schweizerischen Akademie der Medizinischen Wissenschaften (SAMW) in ihre Standesordnung aufzunehmen. Der Text schlägt den Begriff des «unerträglichen Leidens» als Einstiegskriterium für eine Diskussion um die Suizidhilfe vor. Dieses Kriterium ersetzt das bisherige, das forderte, dass die Erkrankung des Patienten die Annahme rechtfertigt, dass das Lebensende nahe ist. Können wir Ärzte Leiden nicht mehr werten, wenn wir dem Patienten gegenüberstehen und fassen wir solches Leiden lieber in numerisch fassbare Begriffe? In der Tat messen wir Schmerz auf einer Skala von 1-10, behandeln Bluthochdruck, Diabetes und Hyperlipidämie nach oft sehr komplizierten Algorithmen als bezifferbare Werte in $\mathrm{mmHg}$ oder $\mathrm{mMol} / \mathrm{l}$ und sprechen in der Folge von non-compliance, wenn diese Werte nicht erreicht werden.

Patienten, die uns in der Praxis aufsuchen, kommen jedoch nicht wegen erhöhten mMol oder $\mathrm{mmHg}$, sondern weil sie leiden. Und seien wir ehrlich: Viele unserer Handlungen basieren nicht auf bezifferbaren Kriterien, sondern auf Beschwerden. Wir ersetzen einen Femurkopf, weil der Patient unerträgliche Schmerzen hat, behandeln eine Prostatahypertrophie, weil der Patient zu viel Mühe beim Urinieren hat, erweitern eine Femoralarterie, weil beim Gehen immer wieder stehen bleiben zu müssen unerträglich wird und verschreiben Medikamente gegen Halluzinationen, wenn der Patient seine Wahrnehmungen nicht mehr aushält.

Leiden ist zwar omnipräsent, und doch scheint es uns Angst zu machen, vor allem, wenn es unerträglich wird. Es ist daher nicht weiter verwunderlich, dass die Medizinliteratur abgesehen von einem ausgezeichneten, bereits im Jahr 1982 erschienenen Text von Eric J. Cassel (1), nicht mit einem Überangebot an Artikeln zur Leidensthematik aufwartet. Erst ab 2002, als der Begriff des unerträglichen Leidens in der EuthanasieGesetzgebung in Holland und Belgien ausdrücklich als Einstiegskriterium eingeführt wurde, begann die Ärzteschaft, darüber nachzudenken [2, 3, siehe auch 4], ganz so, als hätte die Vox populi - vertreten durch die
Parlamentarier dieser Länder - die Ärzteschaft gezwungen, sich erneut mit der Thematik zu befassen. Wie aber wird Leiden definiert? Cassel resümiert: «Suffering is experienced by persons, not merely by bodies, and has its source in challenges that threaten the intactness of the person as a complex social and psychological entity» [1]. Häufig ist nicht einmal der Schmerz die Ursache. In einer qualitativen Studie mit Personen, deren Krebsleiden zuhause behandelt wurde und die nach Euthanasie verlangten, wurden Schwäche, Müdigkeit, Unwohlsein, Abhängigkeit oder Kontrollverlust weitaus häufiger als Grund für unerträgliches Leiden angegeben als medizinische Probleme [2]. Leiden hängt auch von der Vergangenheit der Betroffenen ab, von der Art, wie sie Probleme im Zusammenhang mit körperlichem Verfall angehen, ihrer Lebenserfahrung, ihrem geistigen Leben, ihrer Kultur und natürlich auch von ihrer Umwelt.

Persönlich bedaure ich, dass die Ärztekammer Zahlen als Entscheidungskriterium zur Bewertung einer Suizidbeihilfe bevorzugt, nämlich die noch verbleibende Lebenszeit - sie ist übrigens auch nicht so genau ... und nicht das Leiden, das zwar nicht in Messeinheiten ausgedrückt, vom Patienten jedoch vermittelt und von uns in vertieften und wiederholten Gesprächen nachvollzogen werden kann, wenn wir fähig sind, es zu hören. Die Bevölkerung in Holland, Belgien, Luxemburg und mehreren amerikanischen Bundesstaaten hat dies verstanden. Ihre Ärzteschaft hat das Leiden als Kriterium übernommen, sich dafür eingesetzt und damit insgesamt gute Erfahrungen gemacht. Warum können wir es in der Schweiz nicht ebenso halten? Muss für uns auch das Parlament entscheiden? Sollten wir in diesen Zeiten der Digitalisierung, in denen alle alles messen wollen, nicht vielmehr wieder lernen, Nichtmessbares wahrzunehmen?

Literatur

1 Cassel EJ. The nature of suffering and the goals of medicine. N England J Med 1982;306:639-45.

2 Dees MK, Vernooij-Dassen MJ, Dekkers WJ, Vissers KC, van Weel C. «Unbearable suffering»: a qualitative study on the perspectives of patients who request assistance in dying. Med Ethics 2011;37:727-34

3 Ruijs CDM, Kerkhof AJFM, van der Wal G, Onwuteaka-Philipsen BD: The broad spectrum of unbearable suffering in end-of-life cancer patients. A cross-sectional primary care study in the Netherlands. BMC Palliative Care 2012;11:12.

4 Ausgabe Nr. 1, Band 11 (2018) der Schweizer Zeitschrift für Biomedizinische Ethik Bioethica Forum widmet sich dieser Thematik. 